\title{
ATENCIÓN PRIMARIA EN SALUD Y GESTIÓN URBANA EN COMUNIDADES SUJETAS A ELEVADA FRAGILIDAD SOCIOECONÓMICA Y AMBIENTAL
}

\author{
Felix J. Rosenberg $\mathbb{D}^{1, \mathrm{a}}$, Sonia Carvalho $\mathbb{B}^{1, \mathrm{~b}}$, Marina Rodrigues $\mathbb{( D}^{1, \mathrm{~b}}$, Marcelo Mateus $\mathbb{( D}^{1, \mathrm{~b}}$, \\ Caiett Genial (1) ${ }^{1, c}$ \\ 1 Fórum Itaboraí, Fiocruz, Petrópolis, Brasil \\ a Médico veterinário, máster en Ciencias Médicas; ${ }^{\text {b }}$ asistente social; ${ }^{\mathrm{c}}$ geógrafo.
}

\section{RESUMEN}

Con base en los conocimientos sobre la determinación social de la salud, se plantea el desafío y la estrategia de actuación para fomentar políticas públicas integradas, con foco en el concepto de salud ampliada en los territorios donde prevalecen poblaciones de elevada exclusión social. Para ello, se considera la relación dialéctica existente entre las clases sociales, los territorios y la salud. Partiendo de los objetivos fundadores de la atención primaria de la salud, los equipos de salud de la familia en la municipalidad de Petrópolis, estado de Río de Janeiro, Brasil, fueron capacitados para realizar un diagnóstico rápido participativo relacionado con las metas de los objetivos de desarrollo sostenible, en sus áreas de actuación. Dicho diagnóstico orientó el plan de acción para la promoción de salud en ocho áreas piloto, dentro de las cuales se delimitan conglomerados de mayor exclusión social mediante la cartografía participativa. Siguiendo criterios del control social del sistema de salud, establecidos en la legislación del país, se fomenta la creación de foros comunitarios que elegirán representantes legítimos de la comunidad para integrar los consejos locales de salud, cuya principal misión es discutir los problemas de salud y bienestar a partir de una visión intersectorial y ampliada. Los foros comunitarios, en conjunto con otras organizaciones de la sociedad local, debaten los principales problemas y proponen soluciones alternativas que permitan transformar colectivamente la realidad de los territorios. Como alternativa inicial se establecen proyectos de agricultura urbana de carácter agroecológico para promover transformaciones soberanas y resilientes.

Palabras clave: Atención Primaria de Salud; Determinantes Sociales de Salud; Ciencias Sociales en Salud; Políticas de Control Social; Gestión Ambiental; Monitoreo de Desigualdades en Salud (fuente: DeCS BIREME).

\section{PRIMARY HEALTH CARE AND URBAN MANAGEMENT IN COMMUNITIES SUBJECT TO HIGH SOCIOECONOMIC AND ENVIRONMENTAL FRAGILITY}

Citar como: Rosenberg FJ, Carvalho S, Rodrigues M, Mateus M, Genial C. Atención primaria en salud y gestión urbana en comunidades sujetas a elevada fragilidad socioeconómica y ambiental. Rev Peru Med Exp Salud Publica. 2020;37(2):350-5 doi: https://doi.org/10.17843/rpmesp.2020.372.5796

Correspondencia: Felix Julio Rosenberg; Av. Epitacio Pessoa, 332 / 04. Ipanema, Rio de Janeiro, 22410-090, Brasil; felix.rosenberg@fiocruz.br

Recibido: $14 / 05 / 2020$

Aprobado: 27/05/2020

En línea: $12 / 06 / 2020$

\section{ABSTRACT}

Based on the knowledge of the social determination of health, the challenge and the strategy of action to promote integrated public policies are posed, focusing on the concept of expanded health in territories where populations with high social exclusion prevail. For this purpose, the dialectic relationship between social classes, territories and health is considered. Based on the founding objectives of primary health care, the family health teams in the municipality of Petrópolis, state of Rio de Janeiro, Brazil, were trained to carry out a participatory rapid assessment related to the goals of the sustainable development objectives, in their areas of operation. This diagnosis guided the action plan for health promotion in eight pilot areas, within which conglomerates of greater social exclusion are defined through participatory mapping. Following criteria for the social control of the health system, established in the country's legislation, the creation of community forums is encouraged, which will elect legitimate community representatives to serve on local health councils, whose main mission is to discuss health and welfare problems on the basis of an intersectoral and expanded vision. The community forums, in conjunction with other local society organizations, discuss the main problems and propose alternative solutions that will collectively transform the reality of the territories. As an initial alternative, agro-ecological urban agriculture projects are established to promote sovereign and resilient transformations.

Keywords: Primary Health Care; Social Determinants in Health; Social Sciences and Health; Social Control Policies; Environmental Management; Health Inequality Monitoring (source: MeSH NLM). 


\section{INTRODUCCIÓN}

Durante la última década han incrementado de forma expresiva los conocimientos relacionados con el papel determinante de la estructura social sobre las condiciones de salud y del buen vivir de las comunidades. Muchos de estos conocimientos fueron incorporados en el informe final de la Comisión de Determinantes Sociales de la Salud de la Organización Mundial de la Salud ${ }^{(1)}$ y descritos con detalle por M. Marmot en $2010^{(2)}$. Estos determinantes ya habían sido mencionados por John Snow al estudiar la epidemia de cólera que asoló los países de Europa durante el siglo XIX. Efectivamente, la falta de saneamiento y el hacinamiento urbano constituyen los determinantes críticos para la transmisión de la enfermedad y están relacionados con los barrios habitados por quienes desempeñan las ocupaciones laborales más simples y artesanales ${ }^{(3)}$.

Independientemente de la discusión conceptual que considera, por un lado, las condiciones socioambientales y, por el otro, el modo de producción como determinantes de las inequidades en las condiciones de salud ${ }^{(4)}$, el hecho es que ambas, llamadas de relaciones proximales y distales, respectivamente ${ }^{(5)}$, reflejan las desigualdades sociales que habrán de expresarse en la realidad concreta de un territorio construido y deconstruido permanentemente por sus habitantes ${ }^{(6)}$.

Las posibilidades de intervención para reducir o eliminar las desigualdades sociales y así cambiar la realidad determinante del proceso salud-enfermedad son de extrema dificultad en las condiciones actuales de organización política y económica hegemónica. $\mathrm{O}$ bien requieren una profunda transformación política que lleve a una alteración de las relaciones de poder en nuestras sociedades o bien deben contar con reformulaciones significativas en las políticas y estrategias sectoriales (habitación, educación, saneamiento ambiental, transporte, etc.) que poseen características determinantes sobre las variables ambientales que actúan sobre la salud. Sin embargo, estas políticas suelen actuar en forma vertical, carecen de integración y, consecuentemente, no inciden diferencialmente en la distribución geográfica desigual de las clases y grupos sociales.

De esta forma, el objetivo del trabajo es proponer una estrategia y una metodología que permitan establecer políticas públicas intersectoriales e integradas, con foco en el concepto de salud ampliada, en los territorios donde prevalecen poblaciones de elevada exclusión social, tal como se plantea esquemáticamente en la Figura 1.

\section{TERRITORIO, CLASE SOCIAL Y SALUD}

La caracterización social de los territorios debe ser la primera meta estratégica de cualquier programa público que

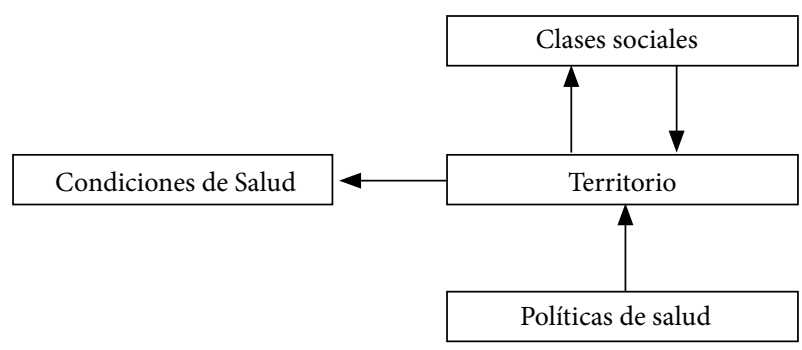

Fuente: Elaboración propia

Figura 1. Clases sociales, territorio y salud

pretenda reducir las desigualdades sociales determinantes de las grandes inequidades en la situación de salud y bienestar. Sin embargo, los datos censales disponibles según espacios geopolíticos predefinidos caracterizan, en general, territorios bastante heterogéneos en cuanto a su composición de clase. Esta heterogeneidad hace que los resultados sean promediales, lo que amortigua las desigualdades territoriales y dificulta el establecimiento de medidas que tienden a su reducción.

Con la finalidad de intentar superar esta dificultad, en 2013 se desarrolló un foro conceptual y técnico en el cual se analizó la relación entre las clases sociales, la ocupación territorial y las condiciones de salud ${ }^{(7)}$. En síntesis, pudo ser confirmado empíricamente que la condición ocupacional, como aproximación de una clase o grupo social, reflejaba mejor los indicadores de salud que algunas variables conocidas como renta o educación ${ }^{(8)}$.

También hubo consenso en cuanto a considerar la salud como el derecho al bienestar/buen vivir de una población, en lugar de partir de indicadores de morbimortalidad o de carga de enfermedad. En este sentido, las condiciones de salud ampliada se aproximan al alcance de la mayoría de las metas de los objetivos del desarrollo sostenible ${ }^{(9)}$.

El principal problema es la cuestión de escala referida a la territorialidad de las clases sociales ${ }^{(10)}$. Cuanto menor la escala, mayor será la heterogeneidad de su estructura social y viceversa. Esto crea el problema de la caracterización de las condiciones de vida de un territorio en una escala que permita representar una hegemonía clara de una determinada clase social. Esta escala casi siempre deberá ser mayor que aquella representada por los menores espacios geográficos para los cuales se disponen de datos secundarios (censales), lo que crea el desafío de la caracterización geográfico-social a partir de la generación de nueva información.

\section{EL CONTROL SOCIAL Y LA ESTRATEGIA DE SALUD DE LA FAMILIA EN BRASIL}

La constitución brasileña de 1988 dedica un importante espacio a la reforma sanitaria, estableciendo la norma cons- 
titucional «Salud, derecho de todos y deber del Estado». A partir de los criterios constitucionales de universalidad, equidad e integralidad en la salud, fue estimulada la atención primaria en salud, que culminaría en 1994 con el Programa de Salud de la Familia, transformado en 2006 en la Estrategia de Salud de la Familia como principal mecanismo para la atención básica en salud ${ }^{(11)}$.

Además de apoyar la función de puerta de entrada de la población al sistema de salud, los agentes comunitarios de salud tienen la responsabilidad de promover la salud y prevenir las enfermedades a través de acciones educativas y de apoyo a individuos, familias y comunidades para implementar prácticas de fomento de vida colectiva y el desarrollo de interacciones sociales ${ }^{(12)}$.

La legislación pertinente al Sistema Único de Salud del Brasil, elaborada de acuerdo con la norma constitucional, establece entre otros, el control social de las políticas y estrategias de salud en los tres niveles de gobierno: federal, estadual y municipal; mediante la creación, en cada nivel, de dos instancias colegiadas de participación comunitaria: las conferencias de salud y los consejos de salud ${ }^{(13)}$.

Las conferencias que ocurren ordinariamente cada cuatro años con elevada representatividad de organizaciones públicas y civiles de representación científica, profesional y ciudadana, fijan las directrices para las políticas sectoriales de salud. Los consejos de salud son órganos colegiados, integrados paritariamente por representantes del gobierno en el nivel correspondiente, trabajadores públicos de la salud y representantes de la sociedad civil. Los consejos de salud están constituidos de forma permanente con carácter deliberativo y su objetivo es formular estrategias y controlar la ejecución de la política de salud.
En 2012, una resolución ministerial reformula la estructura y el funcionamiento de los consejos y menciona, por primera vez, los consejos locales de salud, instancias inframunicipales cuyos detalles de actuación y estructura deben ser establecidos por las propias municipalidades ${ }^{(14)}$. Sin embargo, la constitución y el funcionamiento de estos consejos plantean problemas de no fácil solución. En varias municipalidades de Brasil se ha verificado que los consejeros enfrentan grandes dificultades que desvirtúan los objetivos de participación social por falta de apoyo político, por desconocimiento de sus atribuciones, por incredulidad y por su dependencia de los órganos públicos ${ }^{(16,17,18)}$. Estas dificultades reducen la legitimidad de la representación de los consejeros electos por las comunidades locales ${ }^{(19)}$.

\section{GESTIÓN URBANA Y OBJETIVOS DE DESARROLLO SOSTENIBLE EN LA MUNICIPALIDAD DE PETRÓPOLIS}

La municipalidad de Petrópolis está ubicada a $70 \mathrm{~km}$ de la ciudad de Río de Janeiro, en la región serrana del estado (Figura 2). Petrópolis tiene una población aproximada de 300000 habitantes y se caracteriza por su enorme contraste social y económico, ya que, habiendo sido la sede de verano de la familia imperial, alberga casas y jardines de descanso de algunas de las familias más adineradas de Río de Janeiro y, al mismo tiempo, una elevada población de fuerte exclusión social que ocupa, sobre todo, las laderas de las montañas.

La estructura del nivel primario de atención de la salud está constituida por 44 Unidades de Salud de la Familia, cada

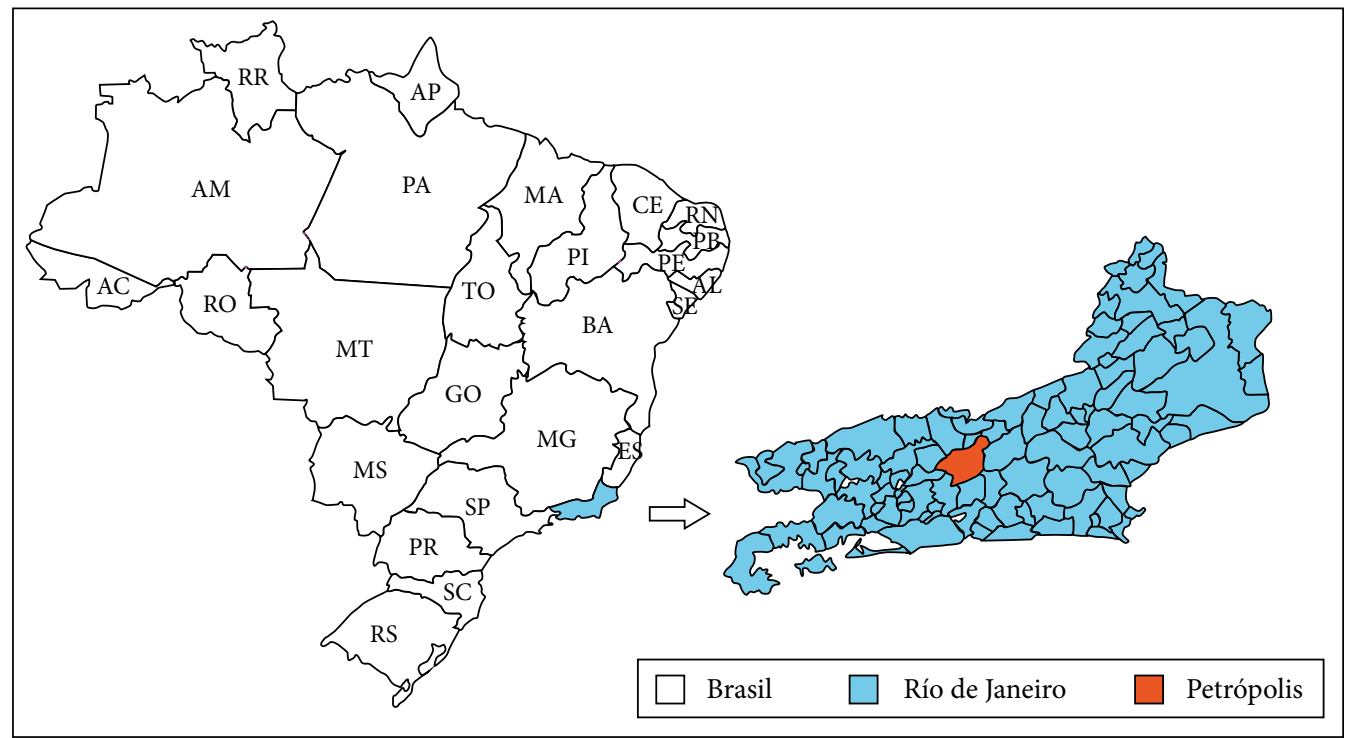

Fuente: Anuario IGEO/UFRJ. Elaboración CEMADEN-RJ

Figura 2. Ubicación de la Municipalidad de Petrópolis 
una de las cuales atiende alrededor de 2600 habitantes, lo que totaliza cerca del $38 \%$ de la población y ocho unidades básicas que atienden al restante del territorio. La ley municipal que crea los consejos locales de salud fue promulgada en $2016^{\left({ }^{(15)} \text {. }\right.}$

Con la finalidad de apoyar la gestión urbana en comunidades más fragilizadas del ámbito municipal, durante el 2017, miembros de 36 de los 44 equipos de salud de la familia realizaron un diagnóstico rápido participativo (DRP) ${ }^{(20)}$ sobre las condiciones de vida y bienestar en sus áreas de cobertura, en escalas que permitiesen caracterizar los aglomerados poblacionales con mayor fragilidad social. La gran ventaja metodológica de la participación de los agentes comunitarios de salud en dichos equipos es que, por el hecho de vivir en esos territorios, gozan de la confianza y la legitimidad necesarias para la incursión científico-técnica.

El trabajo consistió, básicamente, en recorrer el territorio para reconocer características físicas y habitacionales, tales como las áreas de riesgo de accidentes ambientales, vías de comunicación, tipos de viviendas, infraestructura, etc., y conversar con habitantes con los cuales se cruzaban durante los recorridos, sobre sus impresiones relativas a las condiciones de vida y de salud. Los recorridos permitieron también identificar algunos liderazgos locales y habitantes antiguos del barrio, que fueron entrevistados con base en guías semiestructuradas.

Adicionalmente, se organizaron reuniones en los centros de salud, escuelas, iglesias y asociaciones de vecinos con grupos focales de mujeres, con alumnos de cursos para jóvenes y adultos y alumnos del último año de la escuela primaria. Las principales respuestas obtenidas fueron organizadas y sistematizadas de acuerdo con el Objetivo de Desarrollo Sostenible (ODS) al cual estaban referidas. Este trabajo fue analizado por la Conferencia Municipal de Salud de 2017 e incorporado como elemento de la política municipal de promoción de la salud, junto con la creación de los Consejos Locales de Salud (CLS).

Dadas las limitaciones iniciales, tanto metodológicas como de personal, se optó por la realización de un piloto en ocho territorios representativos de las áreas de actuación de los Equipos de Salud de la Familia. El piloto permitió seleccionar, según los resultados del diagnóstico, aquellas áreas consideradas de mayor exclusión social, con la finalidad de investigar con más detalle su realidad y elaborar propuestas de organización comunitaria y gestión urbana que pudieran gatillar transformaciones sociales relevantes.

Los resultados del DRP fueron encuadrados en los siguientes Objetivos de Desarrollo Sostenible (ODS): ODS 1 Erradicación de la pobreza; ODS 2 Hambre cero y agricultura sostenible; ODS 4 Educación; ODS 5 Igualdad de género; ODS 6 Agua limpia y saneamiento; ODS 8 Trabajo y crecimiento económico; ODS 11 Ciudades y comunidades sostenibles; y ODS $16 \mathrm{Paz}$, Justicia e instituciones fuertes, y obviamente el ODS 3 Salud y bienestar para todos. Los detalles de los resultados para cada uno de los ODS se pueden consultar en el Portal del Fórum Itaboraí (21).

Con la finalidad de afinar mejor la escala geográfica que permitiese delimitar con mayor precisión las estructuras urbanas y conformaciones socioespaciales donde prevalecen conglomerados de gran precariedad y desigualdad social y económica se procedió a generar, en cada una de las áreas, mapas diseñados en software abierto ${ }^{(22)}$. Estas delimitaciones fueron realizadas mediante cartografía participativa, construida a partir de visitas al territorio e intervención comunitaria, con destaque particular a grupos focales de estudiantes del último año de la enseñanza primaria y equipos de salud de la familia. Un ejemplo de mapa participativo puede verse en la figura 3.

Realizado el diagnóstico y los cartogramas participativos de cada uno de los territorios piloto, se procedió a apoyar la creación de los consejos locales de salud, con carácter intersectorial. Con el objetivo de darles mayor legitimidad y permitir aproximarlos más a la realidad del territorio, el equipo del Fórum Itaboraí pasó a frecuentar cada una de esas ocho áreas y a invitar a los equipos de salud a analizar con las comunidades de sus microáreas de actuación, los principales problemas identificados. En esa relación próxima, cada localidad eligió sus prioridades y formó una comisión local de vecinos que más tarde se transformó en un foro comunitario, cuya principal atribución inmediata fue la de identificar y elegir a sus miembros más activos y participativos que así adquirían legitimidad para integrar los CLS, como auténticos representantes de la comunidad.

Los foros comunitarios, desarrollados en sesiones mensuales que discuten los principales problemas sociales y de salud que afectan al territorio, preparan de forma efectiva a sus representantes para plantear y discutir dichos problemas en el ámbito de los consejos. Un paso más avanzado de esos foros comunitarios es el fortalecimiento e integración con las asociaciones de vecinos, muchas de las cuales, como se advirtió durante el diagnóstico rápido participativo, estaban desactivadas o poco activas.

El trabajo de diagnóstico comunitario se traduce en la apertura de caminos que conduzcan a una transformación de la realidad percibida. Con ese foco, algunos de los colectivos iniciaron la discusión de alternativas posibles. La agricultura urbana y periurbana ${ }^{(23)}$, de fundamento agroecológico, se destacó como la alternativa de elección por su capacidad de desarrollo sostenible con enorme impacto en la alimentación y la seguridad nutricional, la educación, la economía solidaria y familiar, así como en el refuerzo de las prácticas integrativas de salud y bienestar, al incorporar el cultivo de plantas medicinales.

La utilización de residuos orgánicos para preparar abonos naturales (compostaje) con fines de uso propio o de ven- 


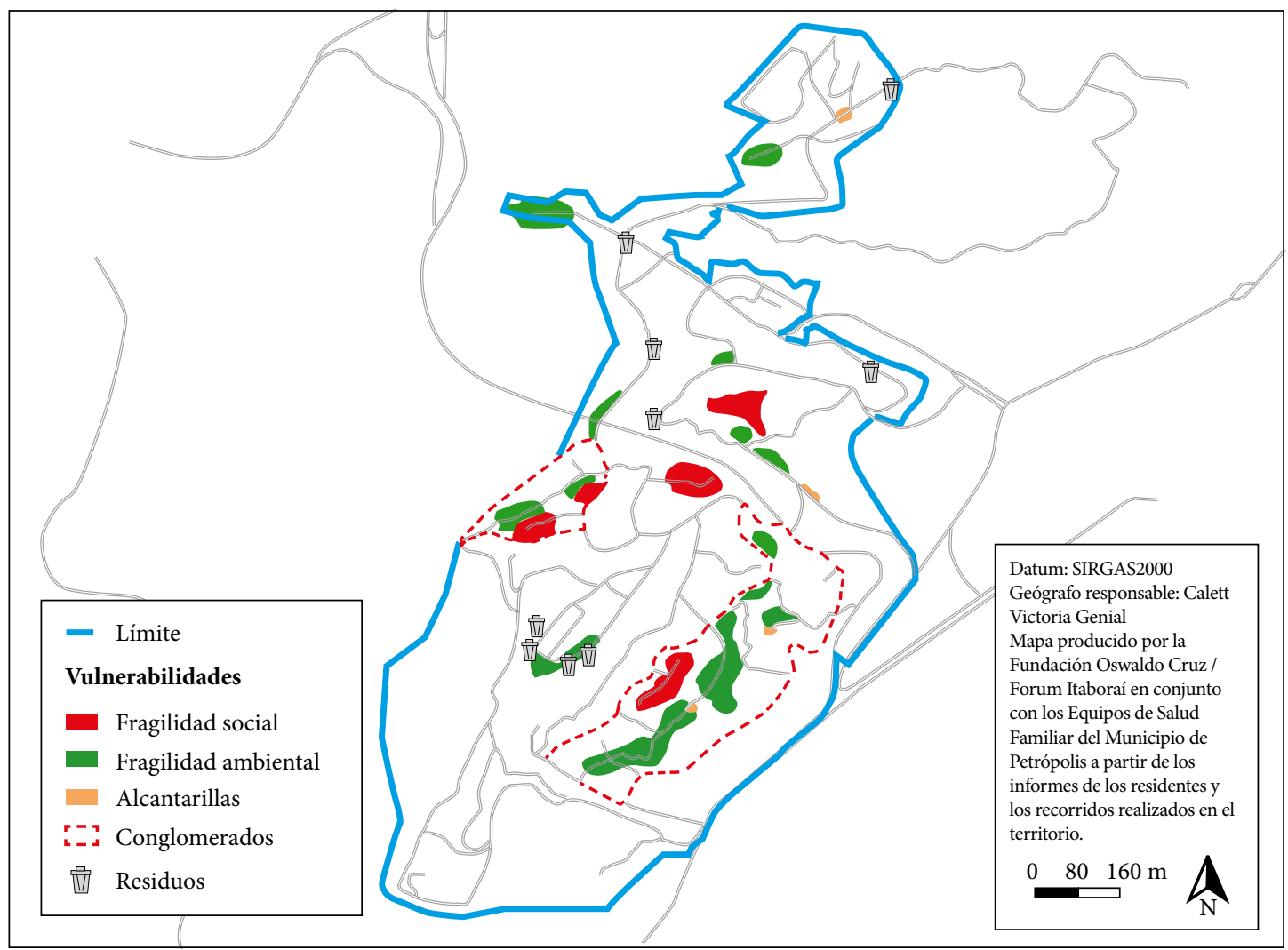

Fuente: Elaboración propia

Figura 3. Mapa de la localidad Amazonas en la escala de alcance de la unidad de salud

ta de excedentes a agricultores de la región, asociada al uso de residuos no biodegradables, como las botellas plásticas, entre otros, para las prácticas de cultivo, contribuyen a la sostenibilidad de la biodiversidad, la conservación y preservación de los recursos hídricos y edáficos y la disminución de los residuos orgánicos y reciclables, aprovechándose mejor los escasos espacios disponibles en dichos territorios. Estas experiencias ya se han iniciado en dos de las áreas piloto, en un caso mediante la constitución de un huerto comunitario y en el otro a través de la creación de huertos familiares, asociados en red.

\section{CONCLUSIONES}

Se concluye que el papel de la atención primaria de la salud, rescatando su concepción original, tiene el potencial de trascender al papel de puerta de entrada para la acción sectorial del sistema de salud y constituirse en uno de los elementos más poderosos del poder público para actuar efectivamente en una transformación territorial que promueva la reducción de las desigualdades sociales, tal como se plantea en los objetivos del desarrollo sostenible.

El trabajo reúne un acúmulo de experiencias y conocimientos para construir un camino posible para la acción pública dirigida a la reducción de las enormes desigualdades sociales y económicas comunes a la realidad del Brasil y de la mayoría de los países de América Latina. La innovación de este trabajo reside en la actuación intersectorial del poder público, centrada en la estructura de la atención primaria de la salud, con participación de la academia e integrada a la comunidad, en escala de conglomerados territoriales de elevada fragilidad social, ambiental y económica. El trabajo no tiene apenas la finalidad de diagnóstico o la investigación, sino tiene el propósito de elaborar propuestas transformadoras de la realidad territorial que le asegure soberanía y resiliencia.

Dadas las dificultades iniciales de la fragmentación de la la gestión pública y las carencias económicas, educacionales, de habitación e infraestructura, el camino aquí trillado no es simple y mucho menos de resultados significativos en el corto plazo. Sin embargo, se apuesta en el desarrollo de una espiral dialéctica que, a medida que acumula nuevas y pequeñas conquistas, fortalece la organización y la participación comunitaria, genera y comparte conocimientos, abre nuevos caminos y, sobre todo, quiebra mitos y culturas fragmentadas que retroalimentan y tienden a consolidar las desigualdades existentes como si fueran una realidad única e inamovible. 


\section{REFERENCIAS BIBLIOGRÁFICAS}

1. World Health Organization (WHO). Closing the Gap in a Generation: Health Equity through Action on the Social Determinants of Health. Final Report of the Commission on Social Determinants of Health. Geneva: WHO; 2008 (citado el 7 de mayo de 2020). Disponible en: https://www. researchgate.net/publication/44847334_.

2. Marmot M, Goldblatt P, Jessica Allen J. Fair Societies and Healthy Life. The Marmot Review. Strategic review of health inequalities in England post-2010. Institute of Health Equity; 2010 (citado el 7 de mayo de 2020). Disponible en: http://www.instituteofhealthequity.org/resources-reports/ fair-society-healthy-lives-the-marmot-review.

3. Snow J. On the Mode of Communication of Cholera. London: John Churchill, Princess Street. Soho. MDCCCXLIX; 1849.

4. Morales-Borrero MC, Borde E, Eslava-Castañeda JC, Concha-SanchezSC. ¿Determinación social o determinantes sociales? Diferencias conceptuales e implicaciones praxiológicas. Rev Salud Pública. 2013; 15(6):797-808.

5. Krieger N. Proximal, Distal, and the Politics of Causation: What's Level Got to Do With It?. Am J Public Health. 2008;98(2):221-30. doi: 10.2105/ AJPH.2007.111278.

6. Haesbaert R. Dos múltiplos territorios à multiterritorialidade, 2004: 20 pp (citado el el 7 de mayo de 2020). Disponible en: http://www.ufrgs.br/ petgea/Artigo/rh.pdf.

7. Fórum Itaboraí: Classes Sociais, Território e Saúde (F. Rosenberg, org). Cadernos do Itaboraí; 2013 (Citado el el 7 de mayo de 2020). Disponible en: http://www.forumitaborai.fiocruz.br/node/896.

8. Figueiredo JA. Class Divisions and Health chances in Brazil. Int J Health Serv. 2011;41(4):691-709. doi: 10.2190/HS.41.4.e

9. CEPAL. The 2030 Agenda and the Sustainable Development Goals An opportunity for Latin America and the Caribbean. Santiago: Publicación de las Naciones Unidas; 2018 (citado el el 7 de mayo de 2020). Disponible en: https://www.cepal.org/sites/default/files/events/files/2030_agenda_and_ the_sdgs_an_opportunity_for_latin_america_and_the_caribbean.pdf.

10. Castro IO. problema da escala. En: Geografia: conceitos e temas. Castro I de, da Costa Gomes PC, Correa RL, Ed. Ed Bertrand Brasil; 1995:117-140.

11. Pinto LF, Giovanella L. Do Programa à Estratégia Saúde da Família: expansão do acesso e redução das internações por condições sensíveis à atenção básica. Ciênc Saúde Colet. 2018; 23(6):1903-13.
12. Mendoça MHM de. Profissionalização e Regulação da atividade do ACS no contexto da reforma sanitária. Trab Educ Saúde. 2004; 2(2):353-65.

13. Presidência da República, Casa Civil, Subchefia para Assuntos Jurídicos. Lei 8142 de 28 de dezembro de 1990 (citado el el 7 de mayo de 2020). Disponible en: http://www.planalto.gov.br/ccivil_03/leis/18142.htm.

14. Ministerio da Saúde, Conselho Nacional de Saúde. Resolución 453 de 10 de maio de 2012 (citado el el 7 de mayo de 2020). Disponible en: http://bvsms.saude.gov.br/bvs/saudelegis/cns/2012/res0453_10_05_2012.html.

15. Prefeitura de Petrópolis. Lei № 7.479 de 27 de dezembro de 2016. Diario Oficial Municipal del 28/12/2016 (citado el el 7 de mayo de 2020). Disponible en:http://www.petropolis.rj.gov.br/pmp/index.php/servicos-na-web/ informacoes/diario-oficial/finish/207-dezembro/3897-5097-quartafeira-28-de-dezembro-de-2016.html.

16. Busana JÁ, Heidemann ITSB, Wendhausen ÁLP. Participação popular em um Conselho Local de saúde: Limites e Potencialidades. Texto contexto - Enferm, 2015; 24(2): 442-9.

17. Lisboa EA, Sodré F, Araújo MD, Quintanilha BC, Luiz SG. Consejos locales de salud: caminos y (des)caminos de la participación social. Trab Educ Saúde 2016;14(3):679-98.

18. Miwa MJ, Serapioni M, Ventura CAA. A presença invisível dos conselhos locais de saúde. Saúde Soc São Paulo. 2017; 26(2):411-23.

19. Oliveira LL, de Souza FÉ, de Ribeiro RS. Conselhos locais de saúde e suas bases: compassos e descompassos. Libertas. 2004-2005; 4-5 (n. Especial): 247-63. doi: 10.34019/1980-8518.2004.v4.18141.

20. Minayo MC de S. Pesquisa social: teoria, método e criatividade. 33ava. ed. Petrópolis: Vozes; 2013.

21. Forum Itaboraí. Síntese dos principais problemas detectados pela comunidade segundo a sua inserção nos Objetivos do Desenvolvimento Sustentável - ODS, Manuscrito, Maio, 2020 (citado el el 7 de mayo de 2020). Disponible en: http://www.forumitaborai.fiocruz.br/node/1069.

22. QuantumGIS ${ }^{\circ}$ (Internet); 2020 (citado el el 7 de mayo de 2020). Disponible en: https://www.qgis.org/pt_BR/site/forusers/download.html.

23. Santandreu A, Lovo IC. Panorama da Agricultura Urbana e Periurbana no Brasil e Diretrizes políticas para a sua promoção, 2007. Belo Horizonte: IPES/RUAF; 2007 (citado el el 7 de mayo de 2020). Disponible en: http:// www.agriculturaurbana.org.br/textos/panorama_AUP.pdf. 